\title{
SOME EQUIVALENT CONDITIONS FOR CONIC SECTIONS
}

\author{
Dong-Soo Kim ${ }^{\mathrm{a}, *}$, Soojeong SeO ${ }^{\mathrm{b}}$, Woo-In Beom ${ }^{\mathrm{c}}$, DeukuU Yang ${ }^{\mathrm{d}}$, \\ JuYEOn KANG ${ }^{\text {e }}$, Jieun JEONG ${ }^{\text {f }}$ AND BoOseOn Song ${ }^{\mathrm{g}}$
}

\begin{abstract}
Let $A$ and $B$ denote a point, a line or a circle, respectively. For a positive constant $a$, we examine the locus $C_{A B}(a)$ of points $P$ whose distances from $A$ and $B$ are, respectively, in a constant ratio $a$. As a result, we establish some equivalent conditions for conic sections. As a byproduct, we give an easy way to plot points of conic sections exactly by a compass and a straightedge.
\end{abstract}

\section{INTRODUCTION}

Straight lines and circles are the best known plane curves, which are characterized as the plane curves of constant curvature. The next most familiar ones are arguably the conic sections.

A circle is also characterized by the fact that the chord joining any two points on it meets the circle at the same angle $([6])$. For a generalization of this property to space curves, see [2]. In this regard, it is interesting to consider what simple geometric properties characterize conic sections ([3], [5]).

A differential geometric characterization of conic sections were studied by the first author and others in terms of the curvature and the support function ([4], [8]).

Let's consider a line $L$ and a point $F$ which is not on $L$. For a positive constant $e$, consider the locus $C_{F L}(e)$ of points $P$ whose distances from $F$ and $L$ are, respectively, in a constant ratio $e$. That is, we consider the locus $C_{F L}(e)=\{P \mid P F=e P L\}$. Then the locus $C_{F L}(e)$ is a conic section with a focus $F$, a corresponding directrix $L$ and eccentricity $e$. More precisely, $C_{F L}(e)$ is a parabola if $e=1$; an ellipse if $0<e<1$; and a hyperbola if $e>1$.

More generally, let $A$ and $B$ denote a point, a line or a circle, respectively. For a positive constant $a$, consider the locus $C_{A B}(a)$ of points $P$ whose distances from

Received by the editors June 1, 2012. Revised August 21, 2012. Accepted September 3, 2012. 2010 Mathematics Subject Classification. 51N24.

Key words and phrases. parabola, ellipse, hyperbola, conic section, directrix, focus.

${ }^{*}$ Corresponding author. 
$A$ and $B$ are, respectively, in a constant ratio $a$. That is, we consider the following locus

$$
C_{A B}(a)=\{P \mid A P=a B P\} .
$$

It is well known that if $A$ and $B$ are distinct points, then the locus $C_{A B}(a)$ is a circle of Apollonius. For the other cases, the following are well-known, or can be easily checked. In our terminology, we state them as follows.

Proposition 1 ([1, p.765], [7, p. 535]). For a line $A$ and a circle $B$ which does not intersect $A$, the locus $C_{A B}(1)$ is a parabola.

Proposition 2 ([1, p.774], [7, p. 542]). For two non-concentric circles $A$ and $B$, suppose that the circle $B$ lies entirely inside $A$. Then the locus $C_{A B}(1)$ is an ellipse.

Proposition 3 ([1, p. 783], [7, p. 550]). For two circles $A$ and $B$ with unequal radii, suppose that $A$ and $B$ do not intersect and that neither circle is inside the other. Then the locus $C_{A B}(1)$ is a branch of a hyperbola.

It is trivial to show that if $A$ and $B$ are distinct lines, then the locus $C_{A B}(a)$ is a single line or the union of two lines.

In this article, in order to find new conditions for conic sections, for a positive constant $a$ we classify the locus $C_{A B}(a)$ for the following three cases, respectively.

1) $A$ is a point and $B$ is a circle.

2) $A$ is a line and $B$ is a circle.

3) $A$ and $B$ are distinct circles.

As a byproduct, at the end of Section 2, we give an easy way to plot points of conic sections exactly.

\section{2. $A$ is a Point and $B$ is a Circle}

In this section, we examine the locus $C_{A B}(a)$ for a point $A$ and a circle $B$. As a result, first of all, we get the following.

Theorem 1. Suppose that $A$ is a point and $B$ is a circle which does not pass through A. For a positive constant a, the locus $C_{A B}(a)$ is as follows.

1) $C_{A B}(1)$ is an ellipse or a branch of a hyperbola according as $A$ lies in the interior of $B$ or not.

2) $C_{A B}(a)(a \neq 1)$ is a circle or the union of two concentric circles centered at the point $A$ if $A$ coincides with the center of $B$. Otherwise, $C_{A B}(a)(a \neq 1)$ 
is not a conic section, but a curve given by a polynomial in $x$ and $y$ of fourth degree.

Proof. Suppose that $B$ is the circle of radius $2 r(r>0)$ centered at $A^{\prime}$ and the distance of $A$ from $A^{\prime}$ is $2 c(c \geq 0)$. We may introduce a coordinate system $(x, y)$ so that $A=(c, 0)$ and $A^{\prime}=(-c, 0)$. For a point $P(x, y)$, by definition, we have $B P=\left|\sqrt{(x+c)^{2}+y^{2}}-2 r\right|$.

Then, we have the following.

$$
\begin{aligned}
& P(x, y) \in C_{A B}(1) \Leftrightarrow A P=B P \\
& \Leftrightarrow c x+r^{2}=r \sqrt{(x+c)^{2}+y^{2}} \\
& \Leftrightarrow\left(r^{2}-c^{2}\right) x^{2}+r^{2} y^{2}=r^{2}\left(r^{2}-c^{2}\right) \text { and } c x+r^{2} \geq 0 \\
& \Leftrightarrow \begin{cases}\frac{x^{2}}{r^{2}}+\frac{y^{2}}{r^{2}-c^{2}}=1, & \text { if } r>c \\
\frac{x^{2}}{r^{2}}-\frac{y^{2}}{c^{2}-r^{2}}=1 \quad \text { and } \quad x \geq r, & \text { if } r<c \\
y=0 \text { and } \quad x \geq-c, & \text { if } r=c\end{cases}
\end{aligned}
$$

This completes the proof of 1$)$. Note that when $r \neq c$, in either case, the conic section $C_{A B}(1)$ has $A$ and $A^{\prime}$ as foci and its eccentricity is given by $e=c / r$.

Now, suppose that $P(x, y)$ lies in the locus $C_{A B}(a)$ with $a \neq 1$. By putting $b=a^{-1}$, we get the following.

$$
y^{2}+x^{2}+\gamma x+\delta=2 \epsilon \sqrt{(x+c)^{2}+y^{2}},
$$

where

$$
\gamma=\frac{2 c\left(1+b^{2}\right)}{1-b^{2}}, \delta=\frac{r^{2}}{1-b^{2}}+c^{2}, \epsilon=\frac{r}{1-b^{2}} .
$$

Hence, by squaring both sides of (2.2) we have

$$
y^{2}=-\left(x^{2}+\gamma x+\delta-2 \epsilon^{2}\right) \pm \sqrt{D / 4}
$$

where

$$
D / 4=4 \epsilon^{2}\left\{(2 c-\gamma) x+\epsilon^{2}-\delta+c^{2}\right\} .
$$

It follows from (2.4) and (2.5) that $C_{A B}(a)$ is not a conic section but a curve of fourth degree, unless $2 c-\gamma=0$. Since $2 c-\gamma=-4 c b^{2} /\left(1-b^{2}\right)$, we see that $2 c-\gamma=0$ iff $c=0$. Hence, $C_{A B}(a)$ with $a \neq 1$ is not a conic section if $c>0$. If $c=0$ (that is, $\left.A=A^{\prime}=(0,0)\right), C_{A B}(a)$ is a circle or the union of two circles centered at $A$.

This completes the proof of Theorem 1. 
Remark 2. Let $A$ be a point. Then a parabola is usually defined as the locus $C_{A B}(1)$ if $B$ is a line not passing through $A$. Similarly, Theorem 1 gives new definitions for the other conic sections as follows.

(1) $C_{A B}(1)$ is an ellipse if $B$ is a circle and $A$ lies in the interior of $B$.

(2) $C_{A B}(1)$ is a branch of a hyperbola if $B$ is a circle and $A$ lies in the exterior of $B$.

Now, with the aid of Theorem 1, we give an easy way to plot points on conic sections. Consider an ellipse $E$ or a branch of a hyperbola $H$ given by, respectively, as follows.

$$
E: \frac{x^{2}}{a^{2}}+\frac{y^{2}}{b^{2}}=1(a>b) \quad \text { or } \quad H: \frac{x^{2}}{a^{2}}-\frac{y^{2}}{b^{2}}=1, x>0 .
$$

Then, the proof of Theorem 1 shows that for the point $A(c, 0)\left(c=\sqrt{a^{2}-b^{2}}\right)$ and the circle $B$ of radius $2 a$ centered at $A^{\prime}(-c, 0)$, the ellipse $E$ is the same as the locus $C_{A B}(1)$ given by (1.1).

For any $P \neq A^{\prime}$, denote by $Q$ the point where the ray $A^{\prime} P$ emanating from $A^{\prime}$ meets the circle $B$. Then the perpendicular bisector of the segment $A Q$ meets the ray $A^{\prime} P$ at a point $P^{\prime}$. It follows from Theorem 1 that $P \in E$ if and only if $P=P^{\prime}$. This suggests an easy way to draw ellipses.

For a branch of hyperbola $H$ in (2.6), we proceed as before. Consider the point $A(c, 0)\left(c=\sqrt{a^{2}+b^{2}}\right)$ and the circle $B$ of radius $2 a$ centered at $A^{\prime}(-c, 0)$. For any $P \in H$, denote by $Q$ the point where the ray $A^{\prime} P$ emanating from $A^{\prime}$ meets the circle $B$. Then, it follows from Theorem 1 that the perpendicular bisector of the segment $A Q$ meets the ray $A^{\prime} P$ at exactly the point $P$. This gives an easy way to draw hyperbolas.

\section{3. $A$ is a Line And $B$ is a Circle}

In this section, we examine the locus $C_{A B}(a)$ for a line $A$ and a circle $B$. As a result, first of all, we get the following.

Theorem 3. Suppose that $A$ is a line and $B$ is a circle. For a positive constant a, the locus $C_{A B}(a)$ is as follows.

1) In case $A \cap B=\phi, C_{A B}(a)$ is a conic section with eccentricity $e=1 / a$.

2) In case $A \cap B$ is a two points set, $C_{A B}(a)$ is the union of two conic sections with eccentricity $e=1 / a$.

3) In case $A \cap B$ is a singleton set, we have three possibilities.

3-1) $C_{A B}(1)$ is the union of a parabola and a half line.

3-2) $C_{A B}(a)(a>1)$ is the union of two ellipses with eccentricity $e=1 / a$. 
3-3) $C_{A B}(a)(a<1)$ is a branch of a hyperbola with eccentricity $e=1 / a$.

Proof. By a suitable choice of coordinates, we may assume that $B$ is the circle of radius $r(r>0)$ centered at the origin $O(0,0)$ and $A$ is the line $x=c(c \geq 0)$. For a point $P(x, y)$, by definition, we have $A P=|x-c|$ and $B P=\left|\sqrt{x^{2}+y^{2}}-r\right|$. By putting $b=a^{-1}$, we obtain the following.

$$
P(x, y) \in C_{A B}(a) \Leftrightarrow B P=b A P
$$

It follows from (3.1) that $C_{A B}(a)$ is contained in the curve given by a polynomial in $x$ and $y$ of fourth degree.

1) If $a=1$, then we have the following.

1-1) In case $A \cap B$ is empty, that is, $c>r$, every point $P(x, y) \in C_{A B}(1)$ satisfies

$$
B P=\sqrt{x^{2}+y^{2}}-r \text { and } A P=c-x .
$$

Hence the locus $C_{A B}(1)$ is given by

$$
y^{2}=-2(c+r) x+(c+r)^{2},
$$

which is a parabola with focus $O$ and directrix $\bar{A}_{+}$defined by $\bar{A}_{+}: x=c+r$.

1-2) If $c=r$, then we have

$$
B P=\left|\sqrt{x^{2}+y^{2}}-r\right| \text { and } A P=|r-x| .
$$

Hence, by squaring $A P=B P$ two times appropriately, we see that the locus $C_{A B}(1)$ is given by

$$
y=0(x \geq 0) \quad \text { or } \quad y^{2}+4 r x-4 r^{2}=0 .
$$

which is the union of a half line and a parabola with focus $O$ and directrix $\bar{A}_{+}$ defined by $\bar{A}_{+}: x=c+r$.

1-3) If $c \in[0, r)$, then we have

$$
B P=\left|\sqrt{x^{2}+y^{2}}-r\right| \text { and } A P=|c-x| .
$$

Hence, by squaring $A P=B P$ two times appropriately, we see that the locus $C_{A B}(1)$ is given by

$$
y^{4}+4 c x y^{2}-2\left(r^{2}+c^{2}\right) y^{2}+4\left(c^{2}-r^{2}\right) x^{2}+4 c\left(r^{2}-c^{2}\right) x+\left(r^{2}-c^{2}\right)^{2}=0,
$$

which can be factored as follows.

$$
\left\{y^{2}-2(r-c) x-(r-c)^{2}\right\}\left\{y^{2}+2(r+c) x-(r+c)^{2}\right\}=0 .
$$

This shows that $C_{A B}(1)$ is the union of two parabolas with common focus $O$ and directrices $\bar{A}_{ \pm}$defined by $\bar{A}_{-}: x=c-r$ and $\bar{A}_{+}: x=c+r$, respectively. 
2) In case $a \neq 1$, we have the following.

$$
P(x, y) \in C_{A B}(a) \Leftrightarrow f(x, y)^{2}-4 r^{2}\left(x^{2}+y^{2}\right)=0 \quad \text { and } \quad f(x, y) \geq 0,
$$

where we denote $f(x, y)=x^{2}+y^{2}+r^{2}-b^{2}(x-c)^{2}$.

It is straightforward to show that the following factorization holds.

$$
f(x, y)^{2}-4 r^{2}\left(x^{2}+y^{2}\right)=\left\{y^{2}+Q(x)\right\}\left\{y^{2}+R(x)\right\},
$$

where

$$
\begin{aligned}
& Q(x)=\left(1-b^{2}\right) x^{2}+2 b(b c+r) x-(b c+r)^{2}, \\
& R(x)=\left(1-b^{2}\right) x^{2}+2 b(b c-r) x-(b c-r)^{2} .
\end{aligned}
$$

Note that for the the lines $\bar{A}_{ \pm}(a)$ defined by $\bar{A}_{ \pm}(a): x=c \pm r / b=c \pm a r$, the following holds.

$$
\begin{aligned}
& y^{2}+Q(x)=0 \Leftrightarrow P(x, y) \in C_{O \bar{A}_{+}(a)}(b), \\
& y^{2}+R(x)=0 \Leftrightarrow P(x, y) \in C_{O \bar{A}_{-}(a)}(b) .
\end{aligned}
$$

Using (3.10) and (3.12), it is straightforward to classify the remaining cases as follows.

2-1) If $c>r$, then $C_{A B}(a)=C_{O \bar{A}_{+}(a)}(b)$ is a conic section with eccentricity $e=b=$ $1 / a$.

2-2) If $c=r$, we have two possibilities.

2-2-1) If $a>1$, then $C_{A B}(a)=C_{O \bar{A}_{+}(a)}(b) \cup C_{O \bar{A}_{-}(a)}(b)$ is the union of two ellipses with eccentricity $e=b=1 / a$.

2-2-2) If $a<1$, then $C_{A B}(a)=C_{O \bar{A}_{+}(a)}(b)$ is a branch of a hyperbola with eccentricity $e=b=1 / a$.

2-3) If $c \in[0, r)$, then $C_{A B}(a)=C_{O \bar{A}_{+}(a)}(b) \cup C_{O \bar{A}_{-}(a)}(b)$ is the union of two conic sections with eccentricity $e=b=1 / a$.

This completes the proof of Theorem 3 .

Remark 4. Suppose that $A$ is a line and $B$ is a circle of radius $r$ centered at $O$. If they do not intersect each other, then the proof of Theorem 3 shows that for a positive constant $a$, the locus $C_{A B}(a)$ coincides with the conic section $C_{O \bar{A}_{+}(a)}(1 / a)$ with eccentricity $e=1 / a$, where $\bar{A}_{+}(a)$ is the parallel displacement of the line $A$ away from $O$ by ar units.

Conversely, for a point $F$ and a line $l$ with $d=F l>0$, we consider a conic section $C_{F l}(b)$ with eccentricity $e=b$. Then, it follows from Theorem 3 that for any $r \in(0, d /(1+a))$ we have $C_{F l}(b)=C_{A B}(a)$ with $a=1 / b$, where $B$ is the circle of 
radius $r$ centered at $F$ and $A$ is the parallel displacement of the line $l$ toward $F$ by ar units.

Remark 5. Theorem 3 shows that the polynomial in $x$ and $y$ of fourth degree given by (3.1) can be factored into the product of two quadratic polynomials in $x$ and $y$.

\section{4. $A$ AND $B$ ARE CIRCles}

In this section, for a positive constant $a$ we examine the locus $C_{A B}(a)$ for two circles $A$ and $B$. If the circles $A$ and $B$ are concentric, then the locus $C_{A B}(a)$ is either a circle or the union of two circles.

Now, we investigate the remaining cases as follows.

Theorem 6. Suppose that $A$ and $B$ are non-concentric circles. We denote by $r$ and $O$ (resp., s and $\left.O^{\prime}\right)$ the radius and center of $A$ (resp., of $B$ ) and we put $d=O O^{\prime}>0$. Then, the locus $C_{A B}(a)$ is as follows.

1) If $a=1$, we may assume that $r \geq s$. Then we have the following.

1-1) In case $d \geq r+s, C_{A B}(1)$ is either a line or a branch of a hyperbola according as $r=s$ or not.

1-2) In case $r-s<d<r+s$, then $C_{A B}(1)$ is either the union of an ellipse $E$ and a branch $H$ of a hyperbola or the union of an ellipse $E$ and a line.

1-3) In case $d=r-s, C_{A B}(1)$ is the union of an ellipse and a ray emanating from the center of the circle $B$.

1-4) In case $d<r-s, C_{A B}(1)$ is an ellipse.

2) If $a>1$, then we have the following.

2-1) In case $r=$ as and $d>r+$ as, the locus $C_{A B}(a)$ is a circle $C_{O O^{\prime}}(a)$.

2-2) In case $r=$ as and $d=r+$ as, the locus $C_{A B}(a)$ is the union of a circle $C_{O O^{\prime}}(a)$ and the center $O^{\prime}$ of the circle $B$.

2-3) Otherwise, the locus $C_{A B}(a)$ is not a conic section.

3) If $a<1$, then we have the following.

3-1) In case $r=a s$ and $a d>r+$ as, the locus $C_{A B}(a)$ is a circle $C_{O O^{\prime}}(a)$.

3-2) In case $r=a s$ and $a d=r+a s$, the locus $C_{A B}(a)$ is the union of a circle $C_{O O^{\prime}}(a)$ and the center $O$ of the circle $A$.

3-3) Otherwise, the locus $C_{A B}(a)$ is not a conic section.

Proof. 1) First, suppose that $a=1$.

If $r=s$, then the perpendicular bisector of $O O^{\prime}$ is always contained in the locus $C_{A B}(1)$. Without loss of generality, we may assume that $r \geq s$. 
1-1) In case $d \geq r+s, A P=O P-r$ and $B P=O^{\prime} P-s$. Hence we get $O P-O^{\prime} P=$ $r-s$. This shows that $C_{A B}(1)$ is either a branch of a hyperbola or a line.

1-2) In case $r-s<d<r+s$, then $C_{A B}(1)$ is the union of an ellipse $E_{O O^{\prime}}(r+s)$ and a branch $H_{O O^{\prime}}(r-s)^{+}$of a hyperbola $H_{O O^{\prime}}(r-s)$ defined by the following, respectively.

$$
E_{O O^{\prime}}(r+s): O P+O^{\prime} P=r+s, \quad H_{O O^{\prime}}(r-s)^{+}: O P-O^{\prime} P=r-s .
$$

Note that when $r=s, H_{O O^{\prime}}(r-s)^{+}$is the perpendicular bisector of $O O^{\prime}$.

1-3) In case $d=r-s, C_{A B}(1)$ is the union of an ellipse $E_{O O^{\prime}}(r+s)$ and the ray emanating from the center of the circle $B$ through the point of tangency.

1-4) In case $d<r-s, C_{A B}(1)$ is an ellipse $E_{O O^{\prime}}(r+s)$.

In order to show that the above classifications exhaust all the points of $C_{A B}(1)$, we proceed as follows. By a suitable choice of coordinates, we may assume that $A$ is the circle of radius $r(r>0)$ centered at the origin $O(0,0)$ and $B$ is the circle of radius $s$ centered at $O^{\prime}(d, 0)$.

Suppose that $P(x, y) \in C_{A B}(1)$. Then we have

$$
\left|\sqrt{x^{2}+y^{2}}-r\right|=\left|\sqrt{(x-d)^{2}+y^{2}}-s\right| .
$$

By squaring the both sides of (4.1) two times appropriately, we see that $P(x, y)$ satisfies

$$
\delta^{2} y^{4}+f(x) y^{2}+g(x)=0
$$

where we use

$$
\begin{aligned}
\delta & =4\left(r^{2}-s^{2}\right), \\
f(x) & =2\left\{\delta\left(\alpha x^{2}+2 \beta x+\gamma\right)-8 r^{2}(2 d x+c)^{2}\right\}, \\
g(x) & =\left(\alpha x^{2}+2 \beta x+\gamma\right)^{2}-16 r^{2}(2 d x+c)^{2} x^{2},
\end{aligned}
$$

and denote

$$
\alpha=4\left(d^{2}+r^{2}-s^{2}\right), \beta=2 d\left(r^{2}+s^{2}-d^{2}\right), \gamma=c^{2}-4 s^{2} d^{2}, c=r^{2}-s^{2}-d^{2} .
$$

Now, it is straightforward to show that the following factorization holds:

$$
\delta^{2} y^{4}+f(x) y^{2}+g(x)=Q(x, y) R(x, y),
$$

where

$$
\begin{aligned}
& Q(x, y)=4(r+s)^{2} y^{2}+4\left\{(r+s)^{2}-d^{2}\right\} x^{2}-4 d\left\{(r+s)^{2}-d^{2}\right\} x-\left\{(r+s)^{2}-d^{2}\right\}^{2}, \\
& R(x, y)=4(r-s)^{2} y^{2}+4\left\{(r-s)^{2}-d^{2}\right\} x^{2}-4 d\left\{(r-s)^{2}-d^{2}\right\} x-\left\{(r-s)^{2}-d^{2}\right\}^{2} .
\end{aligned}
$$


Next, note the following implications:

$$
\begin{aligned}
& O P+O^{\prime} P=r+s \Leftrightarrow Q(x, y)=0, \\
& O P-O^{\prime} P=r-s \Rightarrow R(x, y)=0 .
\end{aligned}
$$

Then, together with (4.5), (4.7) completes the proof of 1) of Theorem 6 .

2) Second, suppose that $a>1$. Then, the similar argument as in the proof of 1 ) shows that

$$
C_{A B}(a)=J \cup K
$$

where

$$
\begin{gathered}
J=\left\{P \mid O P+a O^{\prime} P=r+a s\right\} \cap\left(\left\{P \mid O P \geq r, O^{\prime} P \leq s\right\} \cup\left\{P \mid O P \leq r, O^{\prime} P \geq s\right\}\right), \\
K=\left\{P \mid O P-a O^{\prime} P=r-a s\right\} \cap\left(\left\{P \mid O P \geq r, O^{\prime} P \geq s\right\} \cup\left\{P \mid O P \leq r, O^{\prime} P \leq s\right\}\right) .
\end{gathered}
$$

It follows from the assumption $a>1$ that when $d \geq r+s$, every point $P \in J$ satisfies $O^{\prime} P \leq s$.

Now, it is straightforward to show that $J$ and $K$ are given, respectively, as follows:

$$
J= \begin{cases}\phi, & \text { if } d>r+a s, \\ \text { a point } O^{\prime}, & \text { if } d=r+a s, \\ \text { a curve of quartic polynomial in } x \text { and } y, & \text { otherwise }\end{cases}
$$

and

$$
K= \begin{cases}\text { a circle of Apollonius } C_{O O^{\prime}}(a), & \text { if } r=a s, \\ \text { a curve of quartic polynomial in } x \text { and } y, & \text { otherwise }\end{cases}
$$

Thus, together with (4.10) and (4.11), (4.8) completes the proof of 2) of Theorem 6.

3) Finally, we consider the case $a<1$. Since $C_{A B}(a)=C_{B A}(b)$ with $b=1 / a>1$, the same argument as in the proof of the case $a>1$ shows the following:

$$
C_{A B}(a)=C_{B A}(b)=J^{\prime} \cup K^{\prime},
$$

where

$$
\begin{aligned}
J^{\prime} & =\left\{P \mid O^{\prime} P+b O P=s+b r\right\} \cap\left(\left\{P \mid O^{\prime} P \geq s, O P \leq r\right\} \cup\left\{P \mid O^{\prime} P \leq s, O P \geq r\right\}\right), \\
K^{\prime} & =\left\{P \mid O^{\prime} P-b O P=s-b r\right\} \cap\left(\left\{P \mid O^{\prime} P \geq s, O P \geq r\right\} \cup\left\{P \mid O^{\prime} P \leq s, O P \leq r\right\}\right) .
\end{aligned}
$$


Furthermore, $J^{\prime}$ and $K^{\prime}$ are given, respectively, as follows:

$$
J^{\prime}= \begin{cases}\phi, & \text { if } d>s+b r, \\ \text { a point } O, & \text { if } d=s+b r, \\ \text { a curve of quartic polynomial in } x \text { and } y, & \text { otherwise }\end{cases}
$$

and

$$
K^{\prime}= \begin{cases}\text { a circle of Apollonius } C_{O^{\prime} O}(b)=C_{O O^{\prime}}(a), & \text { if } s=b r \\ \text { a curve of quartic polynomial in } x \text { and } y, & \text { otherwise }\end{cases}
$$

Therefore, (4.12), together with (4.14) and (4.15), completes the proof of 3) of Theorem 6 .

Remark 7. Since a point can be regarded as a circle of radius 0 , Theorem 6 is a generalization of Theorem 1 .

\section{REFERENCES}

1. Anton, H.: Calculus with analytic geometry, 4th ed.. John Wiley and Sons, Inc., New York, 1992.

2. Chen, B.-Y., Kim, D.-S. \& Kim, Y.H.: New characterizations of $W$-curves. Publ. Math. Debrecen 69 (2006), 457-472.

3. Kim, D.-S. \& Kang, S.H.: A characterization of conic sections. Honam Math. J. 33 (2011), no. 3, 335-340.

4. Kim, D.-S. \& Kim, Y.H.: A characterization of ellipses. Amer. Math. Monthly 114/1 (2007), 65-69

5. Kim, D.-S., Kim, Y.H. \& Park, J.H.: Some properties of tangent lines of parabolas. Kyungpook Math. J., to appear.

6. Rademacher, H. \& Toeplitz, O.: The enjoyment of mathematics. Translated from the second (1933) German edition and with additional chapters by H. Zuckerman. Princeton Science Library, Princeton University Press, Princeton, NJ, 1994.

7. Simmons, George F.: Calculus with analytic geometry, 2nd ed.. The McGraw-Hill Companies, Inc., New York, 1996.

8. Yu, Y. \& Liu, L.: A characterization of parabola. Bull. Korean Math. Soc. 45 (2008), no. $4,631-634$.

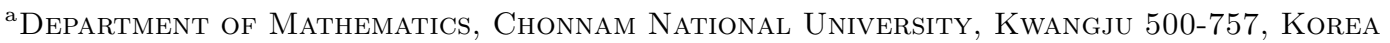
Email address: dosokim@chonnam.ac.kr

${ }^{\text {b}}$ Department of Mathematics, Chonnam National University, Kwanguu 500-757, Korea Email address: soojeongseo98@gmail.com

${ }^{c}$ Department of Mathematics, Chonnam National University, Kwanguu 500-757, Korea Email address: wi-beom@hanmail.net 
${ }^{\mathrm{d} D e p a r t m e n t ~ o f ~ M a t h e m a t i c s, ~ C h o n n a m ~ N a t i o n a l ~ U n i v e r s i t y, ~ K w a n g u u ~ 500-757, ~ K o r e a ~}$ Email address: deukjuyang@gmail.com

e Department of Mathematics, Chonnam National University, Kwanguu 500-757, Korea Email address: juyeonkang81@gmail.com

${ }^{\mathrm{f}}$ Department of Mathematics, Chonnam National University, Kwangju 500-757, Korea Email address: ji-eun93@gmail.com

${ }^{g}$ Department of Mathematics, Chonnam National University, Kwanguu 500-757, Korea Email address: booseons@gmail.com 